\title{
Lymphoepithelioma-like carcinoma of the bladder: a case report
}

\author{
Imad Ziouziou*, Tariq Karmouni, Khalid El khader, Abdellatif Koutani and Ahmed Iben Attya Andaloussi
}

\begin{abstract}
Introduction: Lymphoepitheliomas are malignant epithelial tumors of the nasopharynx characterized by an important lymphoid proliferation at histological examination. Lymphoepithelioma-like carcinoma is a rare tumor of the bladder for which the therapeutic strategy is not clearly defined.

Case presentation: We report the case of a 64-year-old Moroccan man who presented with macroscopic hematuria. Investigations revealed a muscle-invasive lymphoepithelioma-like carcinoma of the bladder. Therefore he underwent a radical cystoprostatectomy with a good outcome.
\end{abstract}

Conclusion: This case illustrates pathogenic, clinical and therapeutic features of this unusual tumor.

Keywords: Bladder, Lymphoepithelioma-like carcinoma

\section{Introduction}

Lymphoepitheliomas are undifferentiated malignant epithelial tumors of the nasopharynx which are recognized histologically by lymphocytic infiltration suggesting an important malignant lymphoma. Tumors of similar histological type have been described in other sites outside of the nasopharynx (lung, stomach, cervix, skin) and are known as lymphoepithelioma-like carcinomas (LELC). The primary urothelial LELC was described for the first time by Zukerberg et al. in 1991 [1]. The LELC is a tumor that rarely reaches the urinary tract: there were only 80 cases reported in the bladder, 10 cases in the ureter, and 7 cases in the renal pelvis [2].

We report the case of a patient who had a muscleinvasive LELC of the bladder, and review the literature on this rare condition in order to clarify the clinical and therapeutic features.

\section{Case presentation}

A 64-year-old Moroccan man had a history of chronic smoking and arterial hypertension treated by losartan and hydrochlorothiazide. He reported a hematuria 2 months ago with blood clots in his urine. His physical examination was normal. Ultrasounds revealed an intravesical echogenic image localized at the left-side wall of his bladder measuring $22 \mathrm{~mm} \times 26 \mathrm{~mm}$ (Figure 1).

Cystoscopic exploration revealed a solid lesion in the left wall with a large base. A transurethral resection of the bladder tumor was performed. A pathological examination revealed a proliferation of cells with large vesicular nuclei, nucleoli and high mitotic activity. These cells were either arranged in syncytial clusters or isolated within a predominant lymphoid stroma. This proliferation infiltrated (Figures 2, 3 and 4) muscle. An immunohistochemical examination (cytokeratin positivity) confirmed the diagnosis of LELC of the bladder (Figure 5).

Chest and abdominopelvic computed tomography (CT) did not show pelvic lymphadenopathy or secondary location. A radical surgery was then decided without neoadjuvant therapy.

Cystoprostatectomy with Bricker diversion and lymph node dissection were performed. The postoperative course was uneventful.

The pathological examination of the surgical specimen revealed no residual tumor lesion and non-invaded lymph nodes (T0 N0).

After 12 months, the patient was in good condition with normal renal function, and normal chest-abdominopelvic CTs at 6 and 12 months.

\footnotetext{
* Correspondence: imad.ziouziou@um5s.net.ma

Service d'Urologie B, Ibn Sina University Hospital, Faculty of Medicine,

Mohamed V Souissi University, Rabat, Morocco
} 


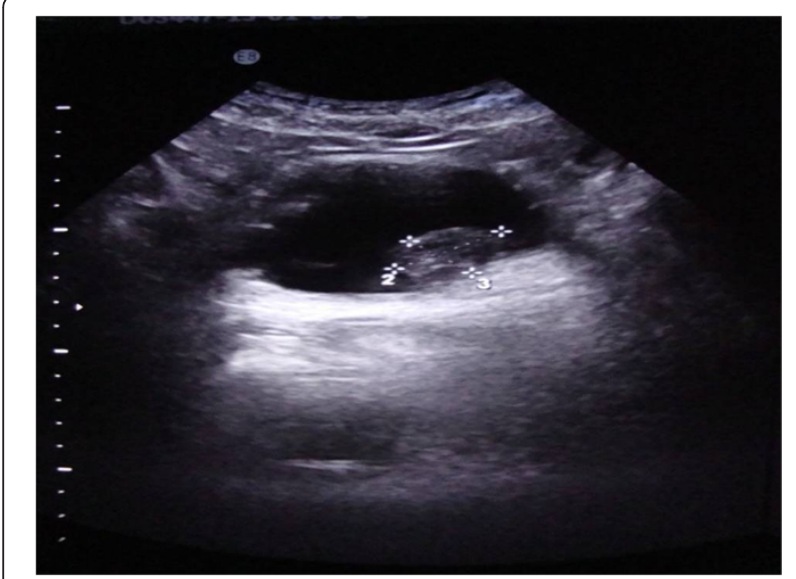

Figure 1 Echogenic ultrasound image at the left wall of the bladder.

\section{Discussion}

LELC of the bladder were classified as a distinct variant of urothelial carcinomas in the classification of the World Health Organization of urothelial tumors in 2004 [3].

The lymphoepithelial carcinoma of the nasopharynx is strongly associated with infection with the Epstein-Barr virus (EBV). However, no risk factor is known for LELC of the bladder [4]; the average age of patients was 65 years with a sex ratio (M:F) of 2:5 [4].

Gulley et al. looked for the presence of EBV in LELC of the bladder by in situ hybridization techniques: none of 11 cases had viral DNA [5].

Two cases of post-Bacillus Calmette-Guérin (BCG) therapy LELC have been reported by Gastaud et al. and Izquierdo-Garcia et al., suggesting a role of immune system activation after the BCG therapy $[6,7]$.

The clinical presentation of LELC of the bladder does not differ from that of urothelial carcinomas. It is

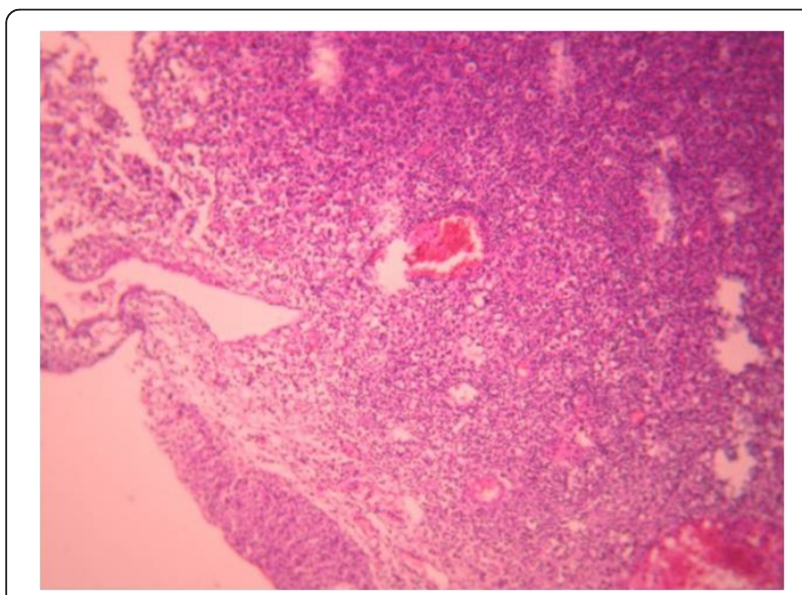

Figure 2 Massive tumoral infiltration of the stroma with regular superficial urothelial mucosa: hematein and eosin $\times 200$.

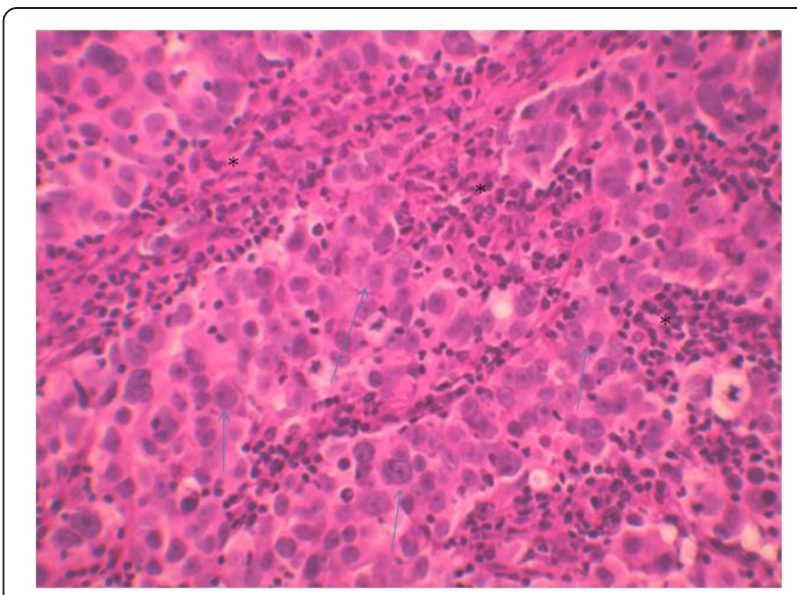

Figure 3 Syncytial clusters of undifferentiated highly vesicular nucleolus nucleus (arrows) cells bathed in abundant lymphoid stroma $(*)$ : hematein and eosin $\times 200$.

dominated by macroscopic hematuria and irritative voiding disorders.

At endoscopy, the tumor is often unifocal, small, and has a polypoid form $[1,2]$. Our case had the same clinical and endoscopic characteristics described in the literature.

LELC is characterized in its pure form by an undifferentiated epithelial tumor with a significant lymphocytic infiltration [8].

Amin et al. subdivided LELC - depending on the importance of urothelial carcinoma within the tumor - into [8]: pure LELC, predominant LELC (lymphoepithelial component greater than 50\%), and focal LELC (lymphoepithelial component less than 50\%).

The predominance of lymphoepithelial component is a good prognostic factor. For some authors, conservative treatment can be achieved even in invasive forms because of their chemosensitivity. Conservative treatment was

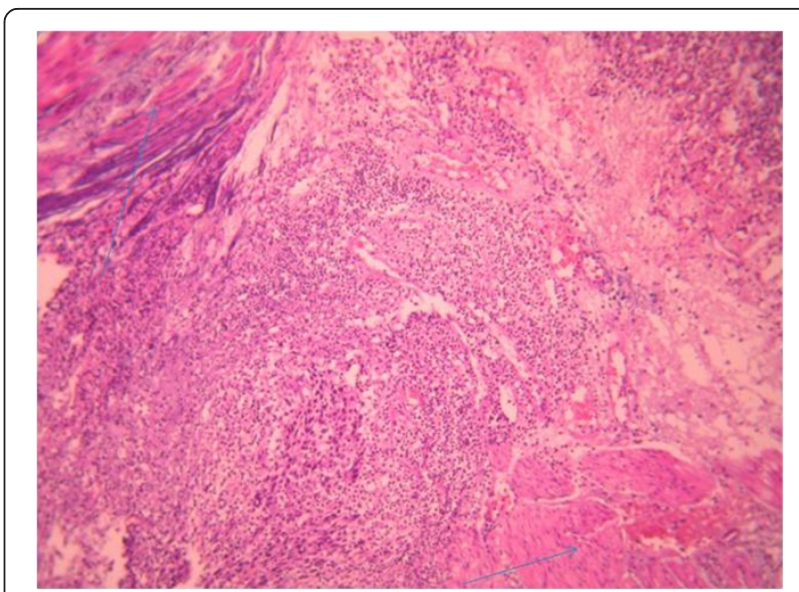

Figure 4 Massive infiltration of muscle (arrows): hematein and eosin $\times 200$. 


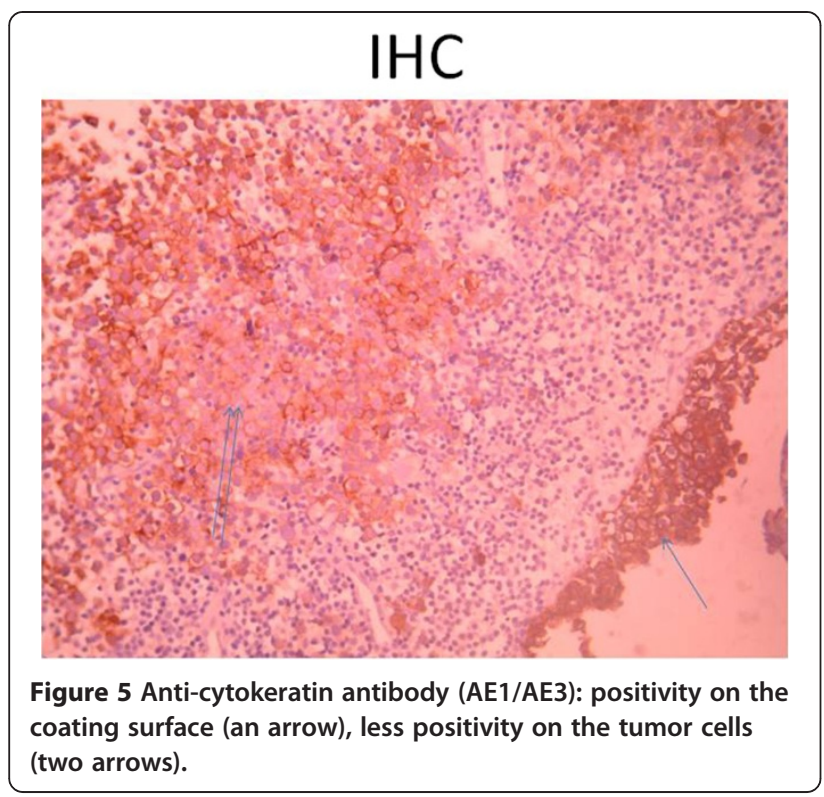

either endoscopic resection or partial cystectomy followed by adjuvant chemotherapy with cisplatin. Progression-free survival was 47 months as reported by Amin et al. [8]. But in this series including 11 patients, only one patient had a predominant LELC form that had chemotherapy with transurethral resection [8]. The number of cases reported and managed by conservative treatment is very low. Most authors recommend radical cystectomy in the case of muscle infiltration [9].

The pathological form of our case was mixed type, in which two components coexisted: lymphoepithelial which was predominant and urothelial.

We opt for radical treatment in patients with urothelial tumor infiltrating the muscle T2 with lymphoepithelial component taking into account the low level of evidence advocating conservative treatment.

Our patient did not have chemotherapy neither neoadjuvant nor adjuvant.

Serrano et al. [4] reported a recurrence-free survival in $87.5 \%$ of patients who had a pure form and stages $\mathrm{T} 2$ or T3. The prognosis of pure forms is linked to the importance of the inflammatory infiltrate and cytotoxic $\mathrm{T}$ lymphocytes for two reasons: early onset of symptoms causing patients to seek care, and strengthening the action of substances used in chemotherapy [4].

Most authors advocate the quasi-systematic use of cisplatin-based chemotherapy as adjuvant treatment after transurethral resection or cystectomy [10]. There are even some that offer BCG therapy [11].

\section{Conclusions}

LELC is a rare tumor of the bladder with no therapeutic consensus. Radical treatment seems most appropriate for muscle invasive urothelial tumors with LELC component, especially as literature data, advocating conservative treatment regardless of the tumor stage, has a low level of evidence.

\section{Consent}

Written informed consent was obtained from the patient for publication of this case report and any accompanying images. A copy of the written consent is available for review by the Editor-in-Chief of this journal.

\section{Abbreviations}

BCG: Bacillus Calmette-Guérin; CT: Computed tomography; EBV: Epstein-Barr virus; LELC: lymphoepithelioma-like carcinoma.

\section{Competing interests}

The authors declare that they have no competing interests.

\section{Authors' contributions}

IZ wrote the paper. TK, KEK, AK, and AIAA contributed by correction of this paper. All the authors read and approved the revised manuscript.

Received: 20 July 2014 Accepted: 26 October 2014 Published: 14 December 2014

\section{References}

1. Zukerberg LR, Harris NL, Young RH: Carcinomas of the urinary bladder simulating malignant lymphoma. Am J Surg Pathol 1991, 15:569e76.

2. Wen SC, Shen JT, Jang MY, Tsai KB, Chang SF, Tsai LJ, Wu WJ: Lymphoepithelial-like carcinoma of ureter - a rare case report and review of the literature. Kaohsiung J Med Sci 2012, 28(9):509-513. doi:10.1016/j.kjms.2012.04.010. Epub 2012 Jul 7.

3. Eble JNSG, Epstein JI, Sesterhenn IA: World Health Organization Classification of Tumours. Tumours of the Urinary System and Male Genital Organs. Lyon: IARC Press; 2004:90e109.

4. Serrano GB, Funez FA, Lopez RG, Crespo CV, Nicolas VD, Naranjo SD, Barrilero ÁE: Carcinoma vesical linfoepiteuoma-like: revision de la literatura y aportacion de un nuevo caso. Arch Esp Urol 2008, 61:723-729.

5. Gulley ML, Amin MB, Nicholls JM, Banks PM, Ayala AG, Srigley JR, Eagan PA, Ro JY: Epstein-Barr Virus is detected in undifferentiated nasopharyngeal carcinoma but not in lymphoepithelial carcinoma of the urinary bladder. Hum. Pathol. 1995, 26:1207-1214.

6. Gastaud O, Demailly M, Guilbert E, Colombat M, Petit J: Le carcinome lymphoépithélial de vessie. Discussion pathogénique. Prog Urol 2002, 12:318-320.

7. Izquierdo-Garcia FM, Garcia-Diez F, Fernandez I, Perez-Rosado A, Saez A, Suarez-Vilela D, Guerreiro-González R: Lymphoepithelial-like carcinoma of the bladder: three cases with clinico-pathological and p53 protein expression study. Virchows Arch 2004, 444:420-425.

8. Amin MB, Ro JY, Lee KM, Ordonnez NG, Dinney CP, Gulley ML, Ayala AG: Lymphoepithelioma-like carcinoma of the urinary bladder. Am J Surg Pathol 1994, 18:466-473.

9. Tamas EF, Nielsen ME, Schoenberg MP, Epstein Jl: Lymphoepithelial-like carcinoma of the urinary tract: a clinicopathological study of 30 pure and mixed cases. Mod Pathol 2007, 20(8):828-834. Epub 2007 Jun 1.

10. Pantelides NM, Ivaz SL, Falconer A, Hazell S, Winkler M, Hrouda D, Mayer EK: Lymphoepithelial-like carcinoma of the urinary bladder: A case report and review of systemic treatment options. Urol Ann 2012, 4(1):45-47. doi:10.4103/0974-7796.91626

11. Fadare O, Renshaw IL, Rubin C: Pleomorphic lymphoepithelioma-like carcinoma of the urinary bladder. Int J Clin Exp Pathol 2009, 2:194-199.

doi:10.1186/1752-1947-8-424

Cite this article as: Ziouziou et al.: Lymphoepithelioma-like carcinoma of the bladder: a case report. Journal of Medical Case Reports 2014 8:424. 